\title{
Indocyanine Green-Loaded Polydopamine-Reduced Graphene Oxide Nanocomposites with Amplifying Photoacoustic and Photothermal Effects for Cancer Theranostics
}

\author{
Dehong $\mathrm{Hu}^{1,2 \dagger}$, Jingnan Zhang ${ }^{1, \dagger}$, Guanhui Gao ${ }^{3}$, Zonghai Sheng ${ }^{1,2}{ }^{\bowtie}$, Haodong Cui ${ }^{1}$, Lintao Cai ${ }^{\bowtie}$ \\ 1. Guangdong Key Laboratory of Nanomedicine, CAS Key Laboratory of Health Informatics, Shenzhen Institutes of Advanced Technology, Chinese Academy \\ of Sciences, Shenzhen 518055, P.R. China; \\ 2. Paul C. Lauterbur Research Center for Biomedical Imaging, Institute of Biomedical and Health Engineering, Shenzhen Institutes of Advanced Technology, \\ Chinese Academy of Sciences, Shenzhen 518055, P. R. China. \\ 3. Paul-Drude Institut, Leibniz-Institut im Forschungsverbund, Hausvogteiplatz 5-7, 10117, Berlin, Germany.
}

tThese authors contributed equally to this work.

$\square$ Corresponding authors: Lintao Cai and Zonghai Sheng; E-mail: 1t.cai@siat.ac.cn. Tel: +86 755 56392210. Fax: +86 75586585222.

() Ivyspring International Publisher. Reproduction is permitted for personal, noncommercial use, provided that the article is in whole, unmodified, and properly cited. See http://ivyspring.com/terms for terms and conditions.

Received: 2015.12.01; Accepted: 2015.12.29; Published: 2016.04.28

\begin{abstract}
Photoacoustic (PA) imaging and photothermal therapy (PTT) as light-induced theranostic platforms have been attracted much attention in recent years. However, the development of highly efficient and integrated phototheranostic nanoagents for amplifying PA imaging and PTT treatments poses great challenges. Here, we report a novel phototheranostic nanoagent using indocyanine green-loaded polydopamine-reduced graphene oxide nanocomposites (ICG-PDA-rGO) with amplifying PA and PTT effects for cancer theranostics. The results demonstrate that the PDA layer coating on the surface of rGO could effectively absorb a large number of ICG molecules, quench ICG's fluorescence, and enhance the PDA-rGO's optical absorption at $780 \mathrm{~nm}$. The obtained ICG-PDA-rGO exhibits stronger PTT effect and higher PA contrast than that of pure GO and PDA-rGO. After PA imaging-guided PTT treatments, the tumors in $4 \mathrm{Tl}$ breast subcutaneous and orthotopic mice models are suppressed completely and no treatment-induced toxicity being observed. It illustrates that the ICG-PDA-rGO nanocomposites constitute a new class of theranostic nanomedicine for amplifying PA imaging and PTT treatments.
\end{abstract}

Key words: Theranostics; Indocyanine green; Reduced graphene oxide; Photoacoustic imaging; Photothermal therapy.

\section{Introduction}

Photoacoustic (PA) imaging and photothermal therapy (PTT) as light-induced imaging and treatment modalities have been attracted much attention very recently.[1-3] PA imaging exhibits high contrast of optical imaging and deep tissue penetration of ultrasound compared to fluorescence imaging.[4-6] Meanwhile, PTT as a noninvasive and effective approach for cancer therapy shows relatively minimal side effects and improving tumor specific killing as compared with traditional therapies.[7] Therefore, the integration of PA imaging and PTT treatment into a single system could provide a novel theranostic platform for cancer diagnosis and therapy. At present, several nanomaterials with excellent near-infared (NIR) light absorption properties and an efficient heat transfer mechanism have been developed as theranostic nanoagents for PA imaging and PTT, including gold nanostructures, $[8,9]$ copper sulfide 
nanoparticle,[11,12] molybdenum disulfide nanosheet, $[13,14]$ carbon nanomaterials, $[15,16]$ polymer nanoparticles.[17-19] Among them, graphene as a typical representative of two-dimensional carbon material has been extensively investigated in many different fields including biomedicine.[20] Functional graphene oxide (GO) and reduced graphene oxide (rGO) show a high specific surface area, and can be used as carriers for both drug and gene deliveries.[21-23] Meanwhile, the intrinsic NIR absorbance allows GO or rGO to be used as PA and PTT agents for in vivo cancer imaging and therapy.[24] Liu et al. reported $\mathrm{rGO}^{-} \mathrm{Fe}_{3} \mathrm{O}_{4}$ nanocomposites as a theranostic agent for fluorescence, PA and magnetic resonance imaging-guided PTT treatments.[25] Our group demonstrated that $\mathrm{rGO}$ could be used as a combined in vivo PA imaging and PTT nanoagent for cancer theranostics in vivo.[26] However, the broad absorption spectrum and low photothermal conversion efficiency of GO or rGO certainly limited their PA imaging sensitivity and phototherapeutic efficiency.

To overcome these limitations, several strategies have been explored to further enhance PA imaging sensitivity and photothermal treatment efficiency in vivo. Yang et al. demonstrated an optimal of size and surface chemistry of rGO could improve its in vivo behaviors, resulting in high tumor retention for PTT.[27] Lim et al. reported a hybrid nanomaterial of rGO anchored gold nanorods for promoting PA imaging in vivo.[28] Recently, Chen et al. developed that the rGO-loaded ultrasmall gold nanorod vesicles could enhance PTT and PA effects for cancer therapy.[29] The combination strategy could not only increase the light absorption efficiency of rGO at the plasmon frequency, but also amplify the PA and PTT performances. Therefore, it is significantly essential to exploit highly enhanced rGO nanocomposites with synergetic light absorption properties and photothermal conversion efficiency.

Herein, we develop a new nanocomposites of indocyanine green (ICG)-loaded polydopamine-rGO (ICG-PDA-rGO) for amplifying PA and PTT effects, and highly promoting cancer theranostics. Remarkably, dopamine, a naturally reduced agent with unique properties of mimicking adhesive proteins, is used to reduce GO by undergoing self-polymerization reaction, and building an adherent polydopamine (PDA) layer coating on the surface of rGO.[30] The coating layer of PDA could improve the water-solubility and biocompatibility of rGO. ICG, a NIR dye approved by the U.S. Food and Drug Administration (FDA),[31,32] is absorbed on the surface of PDA-rGO, resulting in promoting NIR absorption of PDA-rGO for enhancing PA imaging sensitivity and PTT efficiency of cancer.

\section{Materials and methods}

\section{Materials}

Single layer graphene oxide sheets were purchased from Nanjing XFNano Material Tech Co., Ltd (Product No: XF002, Diameter: 1-5 $\mu \mathrm{m}$, Thickness: 0.8-1.2 nm. Dopamine hydrochloride (98\%) and ICG (99\%) were from Sigma-Aldrich. Fetal bovine serum (FBS), trypsin-EDTA solution, Penicillin-streptomycin solution and Roswell Park Memorial Institute 1640 (RPMI 1640) were purchased from Gibco Life Technologies. 3-(4,5-dimethylthiazol-2-yl)-2,5diphenyl tetrazolium bromide (MTT) and dimethyl sulfoxide (DMSO) were purchased from Sigma-Aldrich. Propidium iodide (PI) and calcein-AM were obtained from Invitrogen. All chemicals were used as received without further purification unless otherwise stated. Ultrapure water $(18.25 \mathrm{M} \Omega . \mathrm{cm}$, $25^{\circ} \mathrm{C}$ ) was used to prepare all solutions.

\section{Preparation and characterization of ICG-PDA-rGO}

In a typical process, commercial GO powder $(100$ $\mathrm{mg}$ ) was dispersed in $200 \mathrm{~mL}$ of $10 \mathrm{mM}$ Tris- $\mathrm{HCl}$ solution ( $\mathrm{pH}$ 8.5) under ultrasonication for $5 \mathrm{~h}$ in ice-water bath (a frequency of $20 \mathrm{kHz}$ and power of 130 W VCX130, Sonics, USA), then $50 \mathrm{mg}$ of dopamine hydrochloride was added and dispersed by sonication for $15 \mathrm{~min}$ in an ice bath. The reaction mixture was then stirred at $600 \mathrm{rpm}$ for $12 \mathrm{~h}$ at room temperature. After that, the PDA-rGO was washed, redispersed, and dialyzed in ultrapure water for $72 \mathrm{~h}$. The black powders were dried by freeze drying. To synthesize ICG-PDA-rGO, various mass ratios of PDA-rGO and ICG were prepared to stir overnight in PBS buffer $(\mathrm{pH}$ 7.4) followed by a dialysis in ultrapure water.

\section{Characterizations}

Atomic force microscope (AFM) images were taken using a Nanofirst-3000 AFM. X-ray photoelectron spectroscopy (XPS) measurements were carried out with an ESCALAB 250 high performance electron spectrometer. The ultraviolet-visible (UV-Vis) absorption spectra and fluorescence emission spectra were performed by UV-Vis absorption spectrophotometer (Lambda25, PerkinElmer, USA) and fluorescence spectrophotometer (F900, Edinburgh Instruments, Ltd., U.K.; ex: 740 nm), respectively.

\section{ICG loading efficiency measurements}

To determine ICG loading in PDA-rGO, the ICG-PDA-rGO solution was diluted in $5 \mathrm{~mL}$ of ethyl 
acetate/ethanol (9:1, v/v) and sonicated for $30 \mathrm{~min}$ to extract ICG completely. ICG levels were determined by UV-Vis absorption spectra. ICG loading was defined as ICG content $(\%, w / w)=(I C G$ weight in ICG-PDA-rGO /PDA-rGO weight) $\times 100 \%$. All the measurements were performed in triplicate.

\section{Cells culture}

BEAS-2B normal lung epithelial cells and 4T1 breast carcinoma cells were cultured in RMPI 1640 medium supplemented with $1 \%$ penicillin, $1 \%$ streptomycin, and 10\% heat-inactivated fetal bovine serum (FBS) in a humidified environment of $5 \% \mathrm{CO}_{2}$ at $37{ }^{\circ} \mathrm{C}$.

\section{Animals and tumor model}

Animals received care in accordance with the Guidance Suggestions for the Care and Use of Laboratory Animals. The procedures were approved by Shenzhen Institutes of Advanced Technology, Chinese Academy of Sciences Animal Care and Use Committee. Six-to-seven week old BALB/c mice were maintained under sterile conditions in small animal isolators and were housed in a group of five in standard cages with free access to food and water and a $12 \mathrm{~h} \mathrm{light/dark} \mathrm{cycle.} \mathrm{All} \mathrm{animals} \mathrm{acclimated} \mathrm{to} \mathrm{the}$ animal facility for at least 7 days before experiments. 4T1 breast carcinoma cells were cultured under the standard conditions recommended by American Type Culture Collection. Tumors were generated by subcutaneous injection of $4 \times 10^{6}$ cells in $100 \mu \mathrm{L}$ of PBS onto the back or breast of female BALB/c mice. All possible parameters that may cause social stress, like group size, type (treated and nontreated), etc., among the experimental animals were carefully monitored and avoided. Animals were observed daily for any behavioral abnormalities and weighed weekly.

\section{In vitro PTT}

The PTT effects of ICG-PDA-rGO on 4T1 cells were verified using Calcein $\mathrm{AM}$ and propidium iodide (PI) co-staining. $4 \mathrm{~T} 1$ cells $\left(5 \times 10^{4}\right.$ cells per well) were seeded in 6-well plates and incubated overnight at $37{ }^{\circ} \mathrm{C}$ in a humidified $5 \% \mathrm{CO}_{2}$ atmosphere. After being rinsed with PBS ( $\mathrm{pH}$ 7.4), the cells were incubated with GO, PDA-rGO and ICG-PDA-rGO for $48 \mathrm{~h}$ at $37{ }^{\circ} \mathrm{C}$ respectively under the same conditions. Afterward, the cells of experimental group were rinsed again with PBS and immersed in $200 \mu \mathrm{L}$ of fresh culture medium, and subsequently illuminated using an $808 \mathrm{~nm}$ laser with energy density of 0.6 $\mathrm{W} / \mathrm{cm}^{2}$ for $5 \mathrm{~min}$. After another $12 \mathrm{~h}$ incubation cells were stained with calcein-AM for visualization of live cells and with PI for visualization of dead/late apoptotic cells, according to the manufacture's suggested protocol (Invitrogen). Then, the cells of experimental group were rinsed again with PBS and were examined with biological inverted microscope (Olympus IX71, JPN).

The PTT effects of ICG-PDA-rGO on 4T1 cells were further verified by MTT. 4T1 cells $\left(1 \times 10^{4}\right.$ cells per well) were seeded in 96-well plates and incubated overnight at $37{ }^{\circ} \mathrm{C}$ in a humidified $5 \% \quad \mathrm{CO}_{2}$ atmosphere. After being rinsed with PBS ( $\mathrm{pH} 7.4)$, the cells were incubated with GO, PDA-rGO and ICG-PDA-rGO for $48 \mathrm{~h}$ at $37{ }^{\circ} \mathrm{C}$ under the same conditions. Afterward, the cells of experimental group were rinsed again with PBS and immersed in $200 \mu \mathrm{L}$ of fresh culture medium, and subsequently illuminated using an $808 \mathrm{~nm}$ laser with energy density of $0.6 \mathrm{~W} / \mathrm{cm}^{2}$ for $5 \mathrm{~min}$. The laser spot was adjusted to fully cover the area of each well. After NIR laser illumination, cells were incubated for $12 \mathrm{~h}$ in a $5 \%$ $\mathrm{CO}_{2}, 95 \%$ air humidified incubator at $37{ }^{\circ} \mathrm{C}$. Dark control group was kept under identical conditions as the experimental group except for illumination. The standard MTT assay was carried out to evaluate the cell viability.

\section{Infrared Thermal and PA imaging}

BALB/c mice with $4 \mathrm{~T} 1$ ectopic transplantation tumor and orthotopic transplantation tumor were used as the animal modal. The imaging experiments were performed when tumors reached $\sim 50 \mathrm{~mm}^{3}$. The mice were intratumoral injected with PBS, GO, PDA-rGO and ICG-PDA-rGO $(\mathrm{n}=3)$. The tumors were irradiated by the $808 \mathrm{~nm}$ laser at $0.6 \mathrm{~W} / \mathrm{cm}^{2}$ for 5 min. Region temperatures and infrared thermographic maps were obtained with an infrared thermal imaging camera (Ti27, Fluke, USA). In vivo PA imaging was obtained with preclinical PA computerized tomography scanner (Endra Nexus 128).

\section{In vivo $\mathrm{PTT}$}

For PTT studies, 35 mice bearing 4T1 tumors were randomly divided into seven groups. The treatment scheme is as follows: (a) PBS-laser; (b) GO; (c) PDA-rGO; (d) ICG-PDA-rGO; (e) GO-laser; (f) PDA-rGO-laser; and (g) ICG-PDA-rGO-laser. When the tumor size reached about $50 \mathrm{~mm}^{3}$, the photoirradiation was applied after the intratumoral injection of PDA-rGO, ICG-PDA-rGO, or free ICG $\left(808 \mathrm{~nm}, 0.6 \mathrm{~W} / \mathrm{cm}^{2}\right.$ for $\left.5 \mathrm{~min}\right)$. The spot of laser beam was adjusted to cover the entire region of tumor. The tumor sizes and body weights were inspected every 3 days. The tumor weight was estimated using the formula, tumor volume $=$ length $\times(\text { width })^{2} / 2$, assuming a tumor density of $1 \mathrm{mg} / \mathrm{mL}$. The volume of tumors was evaluated by normalizing the measured values. After therapy, major organs as well as tumors 
were collected and sectioned to $8 \mu \mathrm{m}$ slices for $\mathrm{H} \& \mathrm{E}$ staining.

\section{Histology evaluation}

$\mathrm{H} \& \mathrm{E}$ staining was performed according to a protocol provided by the vendor (BBC Biochemical). Briefly, $8 \mu \mathrm{m}$ cryogenic slides were prepared and fixed with $10 \%$ formalin for about $30 \mathrm{~min}$ at room temperature. After washing with running water for 5 min, the slides were treated with gradient concentrations of alcohol $(100 \%, 95 \%$, and $70 \%)$, each for $20 \mathrm{~s}$. The hematoxylin staining was performed for about $3 \mathrm{~min}$ and washed with water for $1 \mathrm{~min}$. The eosin staining was performed for about $1 \mathrm{~min}$. The slides were washed, treated with xylene, and mounted with Canada balsam. The images were acquired on a Nikon Eclipse 90i microscope.

\section{Results and Discussion}

\section{Synthesis and characterization of ICG-PDA-rGO}

The preparation process of ICG-PDA-rGO was described in Figure 1a. Dopamine monomers were loaded on the surface of commercial GO, and spontaneously self-polymerize via Michael addition/Schiff reaction to form PDA layer coating on the surface of rGO.[33] The free ICG dyes were absorbed on the surface of PDA-rGO via hydrogen bond and $\pi-\pi$ stacking interactions. Atomic force microscope (AFM) imaging showed that the size of commercial GO was less than $1.0 \mu \mathrm{m}$, and the thickness of GO was $0.86 \mathrm{~nm} \pm 0.02 \mathrm{~nm}$ (Figure $1 \mathrm{~b}$ and 1c). After reduction process, the size of ICG-PDA-rGO was not changed obviously, however, the thickness increased to over $2 \mathrm{~nm}$ (Figure $1 \mathrm{e}$ and 1f). The increased thickness of GO proved that PDA coated on surface of rGO. XPS spectra analysis illustrated that the values of the C-C $(284.8 \mathrm{eV})$ and C-O $(286.8 \mathrm{eV})$ decreased, and a new peak of C-N $(285.8 \mathrm{eV})$ was observed (Figure 1d and 1g), further demonstrating the conversion from GO to PDA-rGO.[34] The ICG loading efficiency of $5.8 \%$ to $25.6 \%$ was achieved by adjusting the weight ratio of ICG/ PDA-rGO (Figure S1). The UV-Vis-NIR spectra showed the characteristic absorption peak of ICG-PDA-rGO was located at $780 \mathrm{~nm}$, and the absorbance of ICG-PDA-rGO increased about 13 folds comparing with that of PDA-rGO, further exhibiting successful loading of ICG onto PDA-rGO (Figure 2a). Meanwhile, the fluorescence intensity of ICG-PDA-rGO at $810 \mathrm{~nm}$ decreased by $85.7 \%$ compared to the equal amount of free ICG (Figure 2b). The enhanced NIR absorption and decreased fluorescence emission of ICG-PDA-rGO were likely owing to the direct contact between ICG and PDA-rGO nanosheet and fluorescence resonance energy transfer, which was benefited for translating the light to thermal expansion/heat for PA imaging and PTT treatments by nonradioactive decay. (a)

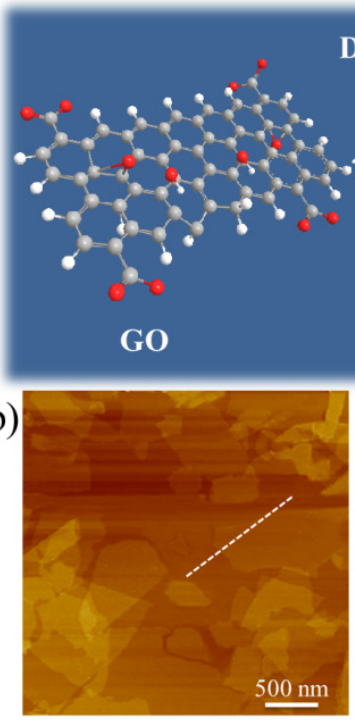

(e)

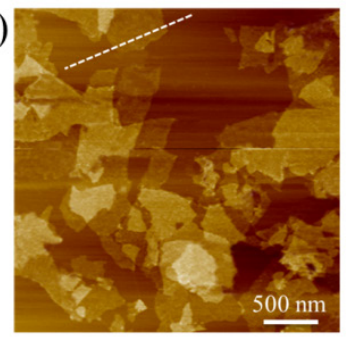

Dopamine

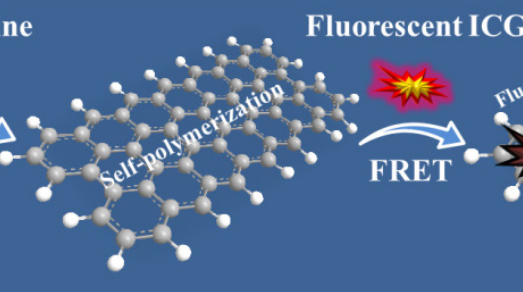

PDA-rGO
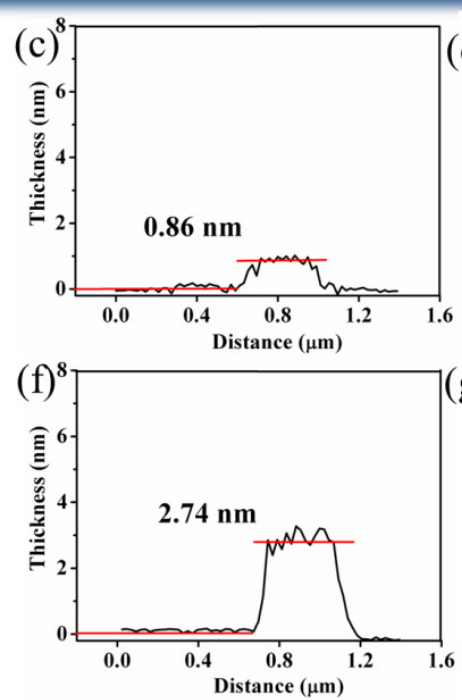
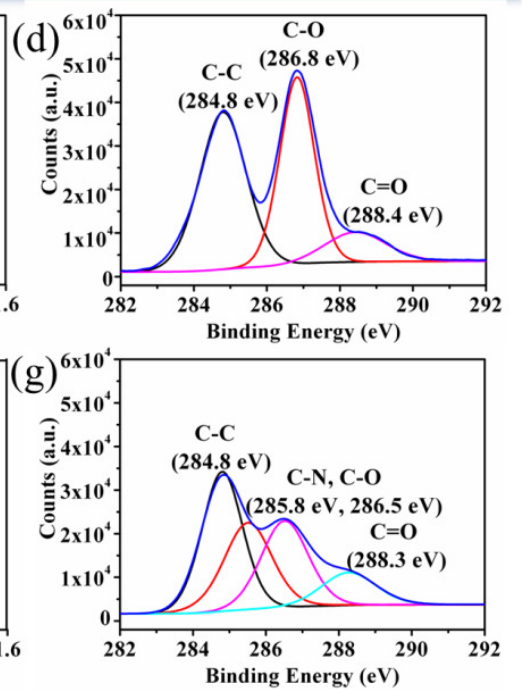

Figure 1. Synthesis and characterization of ICG-PDArGO. (a) Schematic illustration of the prepared process of ICG-PDA-rGO. AFM images of GO (b) and ICG-PDA-rGO (e). The AFM height analysis of GO (c) and ICG-PDA-rGO (f). The $\mathrm{C}_{\mathrm{Is}}$ XPS analysis of $\mathrm{GO}$ (d) and ICG-PDA-rGO (g). 


\section{In vitro PTT effect of ICG-PDA-rGO}

The enhanced NIR absorption of ICG-PDA-rGO allowed for effective photothermal nanoagent for PTT. As shown in Figure 3a, under the same concentration $(200 \mu \mathrm{L}$ of solution with $20 \mathrm{mg} / \mathrm{L})$ and laser irradiation $\left(808 \mathrm{~nm}, 0.6 \mathrm{~W} / \mathrm{cm}^{2}, 5 \mathrm{~min}\right)$ conditions, the temperature of GO, PDA-rGO and ICG-PDA-rGO reached $33.7^{\circ} \mathrm{C}, 40.2^{\circ} \mathrm{C}$ and $54.4^{\circ} \mathrm{C}$, respectively. The ICG-PDA-rGO exhibited more effective than pure GO, PDA-rGO respectively, and previously reported GO derivatives in terms of photothermal heating under the NIR laser irradiation.[35] The photothermal effect of ICG-PDA-rGO was concentration-dependent (from $2.5 \mathrm{mg} / \mathrm{L}$ to $20 \mathrm{mg} / \mathrm{L}$ ), and the temperature increased monotonically with the increasing of ICG-PDA-rGO concentration (Figure 3b). Moreover, ICG-PDA-rGO showed higher stability than that of free ICG after continuous laser irradiaton for 4 cycles (Figure 3c). During the process, the color and absorption spectra of ICG-PDA-rGO were not changed obviously (Figure $3 \mathrm{~d})$. On the contrary, the serious photobleaching of free ICG was observed, and the absorbance decreased by $68 \%$ at the wavelength of $780 \mathrm{~nm}$ and the color of ICG solution changed from light green to brown. The results indicated that the loading ICG onto PDA-rGO enhanced PTT performance and improved the photostability of ICG, which was favorable to promote PTT in vitro.
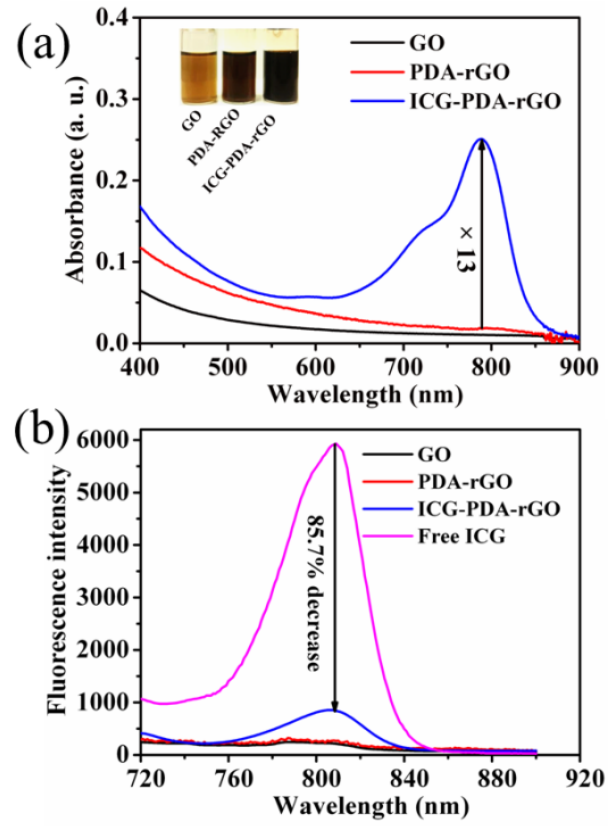

Figure 2. The optical properties of ICG-PDA-rGO. (a) UV-vis absorption spectra of GO, PDA-rGO and ICG-PDA-rGO. The inset shows photographs of GO, PDA-rGO and ICG-PDA-rGO aqueous solutions. (b) Fluorescence spectra of GO, PDA-rGO, ICG-PDA-rGO and free ICG. The arrow indicates that the fluorescence of free ICG decreased by $85.7 \%$ after loading on the surface of PDA-rGO.
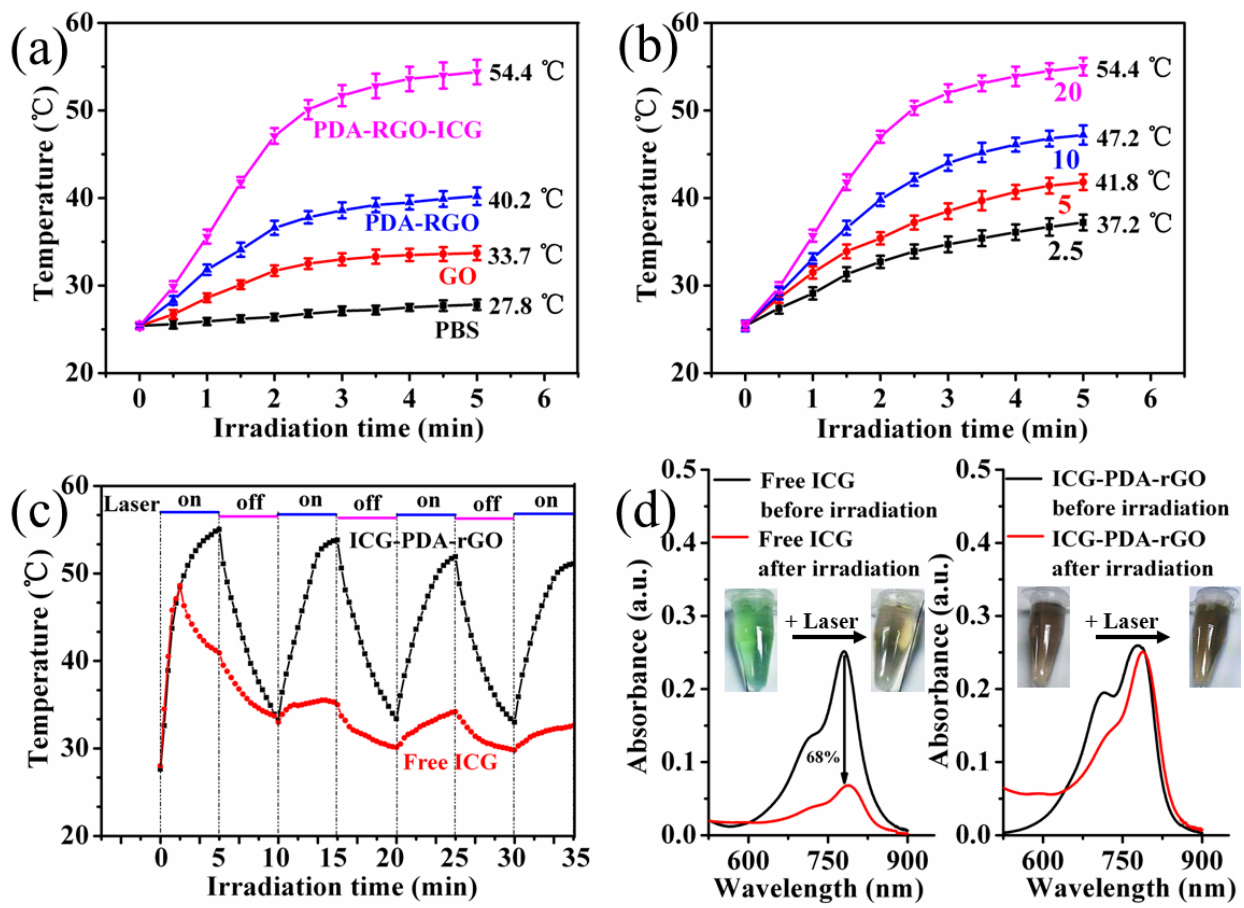

Figure 3. The photothermal effect of ICG-PDA-rGO. (a) Photothermal heating curves of PBS, GO, PDA-rGO and ICG-PDA-rGO solutions. $C_{G O}=C_{P D A}-r G O=C_{I C G-P D A-r G O}=20$ $\mathrm{mg} / \mathrm{L}$. (b) Photothermal heating curves of ICG-PDA-rGO with different concentrations. From down to up: 2.5, 5, 10, 20 mg/L. (c) Temperature evaluation of ICG-PDA-rGO and equal free ICG solutions over four laser ON/OFF cycles. (laser ON time: 5 min, laser OFF time: 5 min). (d) The UV-vis absorption spectra of free ICG and ICG-PDA-rGO solutions before and after four cycles of laser irradiation. The insets show the photographs of free ICG and ICG-PDA-rGO solutions before and after four cycles of irradiation. The laser power was $808 \mathrm{~nm} @ 0.6 \mathrm{~W} / \mathrm{cm}^{2}$. 


\section{In Vitro PA performance of ICG-PDA-rGO}

Next, the PA imaging performance of the free pure GO, PDA-rGO and ICG-PDA-rGO were investigated using Nexus 128 preclinical PA imaging system with a $780 \mathrm{~nm}$ plus laser as an excitation light source. The NIR excited wavelength showed negligible tissue scattering and maximal penetration depth. The laser fluency at the wavelength of $780 \mathrm{~nm}$ was $9 \mathrm{~mJ} / \mathrm{cm}^{2}$ per pulse, which is well below the 20 $\mathrm{mJ} / \mathrm{cm}^{2}$ ANSI laser safety limit.[36] As shown in Figure 4a, pure GO solution exhibited relatively weak PA signal, and the PDA-rGO solution generated moderate PA signal. The ICG-PDA-rGO generated the strongest PA signals about 20 times than that of GO under the same conditions. The signal intensity was dependent on the concentration of the ICG-PDA-rGO (Figure 4b). Insert showed that the PA signals increased with increasing of the concentration of ICG-PDA-rGO, and the linear range is $0.5-4.0 \mathrm{mg} / \mathrm{L}$.
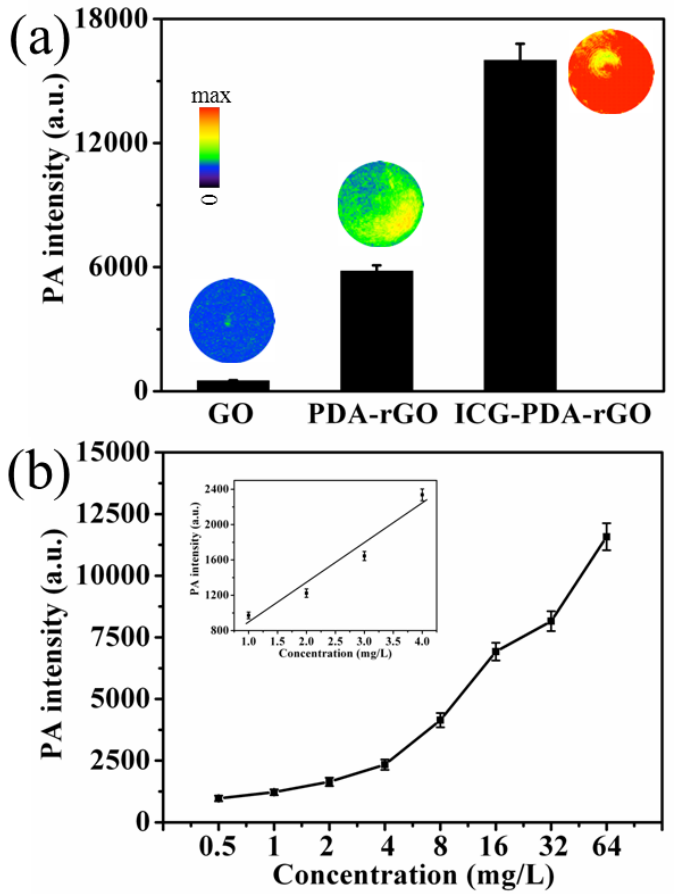

Figure 4. The optical PA imaging of ICG-PDA-rGO solution. (a) PA signal intensity and PA images of GO, PDA-rGO and ICG-PDA-rGO aqueous solutions. $\mathrm{C}_{G O}=\mathrm{C}_{\mathrm{PDA}-\mathrm{rGO}}=\mathrm{C}_{\text {ICG-PDA-rGO }}=10 \mathrm{mg} / \mathrm{L}$. (b) The relationship between PA signal intensity and the concentration of ICG-PDA-rGO. Insert shows the linear relationship between PA signal intensity and the concentration of ICG-PDA-rGO.

\section{In vitro PTT}

To evaluate the cytotoxicity of the prepared GO derivatives, 4T1 breast cancer cells and human BEAS-2B normal bronchial epithelial cells were incubated with GO, PDA-rGO and ICG-PDA-rGO, respectively, at different concentrations for $48 \mathrm{~h}$. The relative viabilities were determined by standard methyl thiazolyl tetrazolium (MTT) assay. No obvious cytotoxicity was observed in all treated groups, indicating the as-prepared ICG-PDA-rGO was biocompatible (Figure 5a and 5b). The ICG-PDA-rGO in cancer cells could induce local hyperthermia for killing cancer cells under the $808 \mathrm{~nm}$ NIR laser irradiation. In order to visually evaluate the in vitro therapeutic effect of ICG-PDA-rGO, the cells were stained with calcein-AM and PI to identify live and dead/late apoptotic cells, respectively. As shown in Figure $5 c$, cells all displayed green fluorescence in the control group, suggesting no effect of pure laser irradiation alone on 4T1 cells. In the GO and PDA-rGO treated groups, some cells were killed and displayed red fluorescence. The ICG-PDA-rGO treated group induced most of cells death and exhibited intense red fluorescence. The quantitative results of MTT assay showed that the GO, PDA-rGO and ICG-PDA-rGO treated groups induced $8.4 \%$, $32.7 \%$ and $92.8 \%$ of $4 \mathrm{~T} 1$ cell dead, respectively (Figure $5 \mathrm{~d})$. The results were in a good agreement with the visual imaging, which indicated a superior photothermal ablation effect of ICG-PDA-rGO in 4T1 cells.

\section{In vivo $P A$ imaging}

In present study, we used intratumoral (i.t.) delivery of ICG-PDA-rGO for the assessment of the PA imaging ability of GO derivatives in 4T1 tumor-bearing mice (Figure 6a). The reason for using i.t. injection is the limitation of ICG-PDA-rGO's lateral dimension $(<1.0 \mu \mathrm{m})$. However, the local delivery method also could be used to investigate the PA effects of different GO derivatives in vivo, which has been emerging as an effective imaging and treatment tool for many types of localized operable and inoperable solid tumors due to the high imaging sensitivity and low systemic toxic side-effects.[37,38] As shown in Figure 6b, the PBS-treated group showed low PA signal, and the blood vessels in tumor area were observed owing to the existence of hemoglobin as an endogenous contrast agent.[39] The ICG-PDA-rGO-treated group showed stronger PA signal in tumor than that of GO and PDA-rGO-treated groups (Figure $6 \mathrm{~b}$ ). Quantitative results indicated that the PA signals of ICG-PDA-rGO in tumor increased approximately 4 times as compared to GO and PDA-rGO-treated groups (Figure 6c). It indicated the high PA imaging sensitivity of the prepared ICG-PDA-rGO in tumor. 

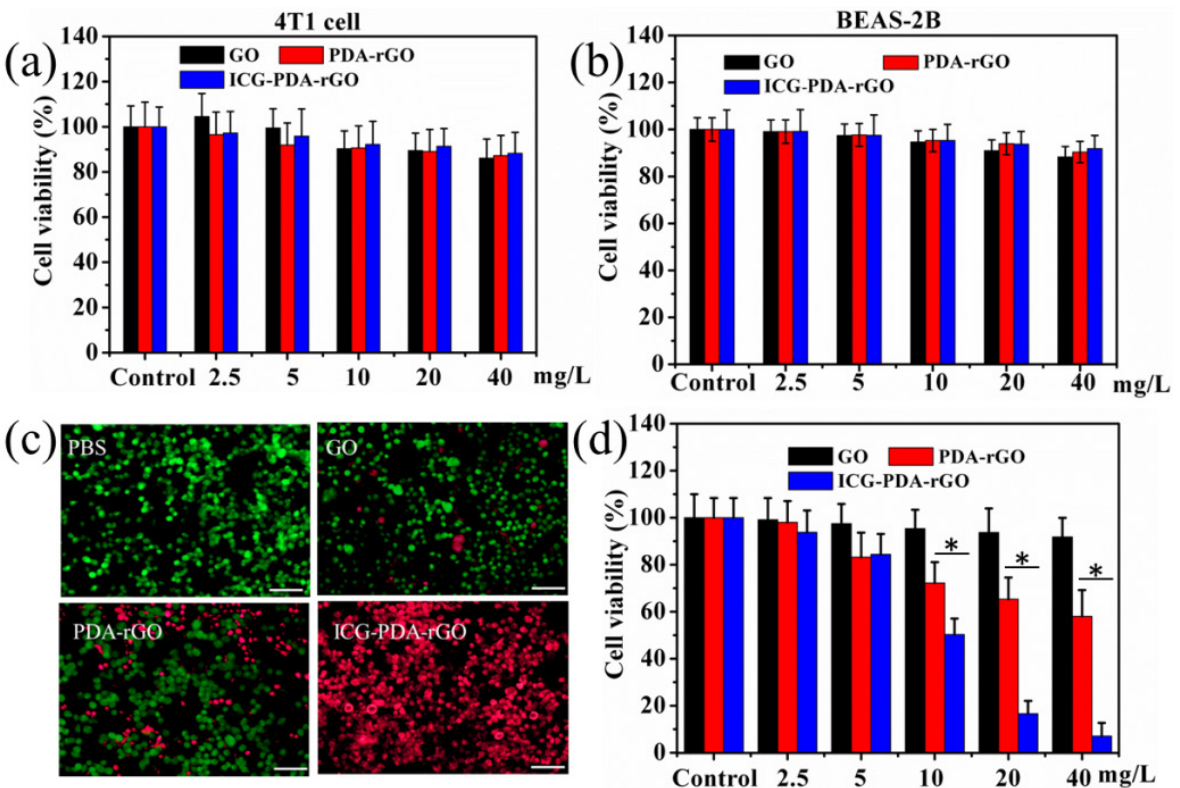

Figure 5. Cytotoxicity and in vitro PTT. Relative viability of $4 \mathrm{TI}$ cells (a) and BEAS-2B cells (b) incubated with various concentrations of GO, PDA-rGO and ICG-PDA-rGO for 48h. (c) Fluorescence images of Calcein AM/PI stained 4T1 cells incubated with PBS, GO, PDA-rGO and ICG-PDA-rGO. Scale bars are 50 $\mu$ m. (d) Quantitative detection of 4T1 cells viability following PTT with ICG-PDT-rGO for $5 \mathrm{~min}$. Laser irradiation dose: $808 \mathrm{~nm}, 0.6 \mathrm{~W} / \mathrm{cm}^{2}, 5 \mathrm{~min} .(*) \mathrm{P}<0.05$.
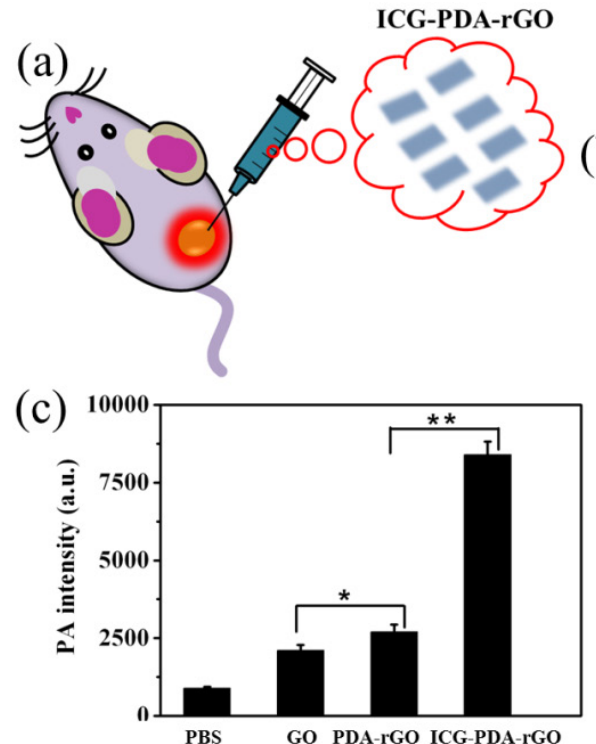

(b)
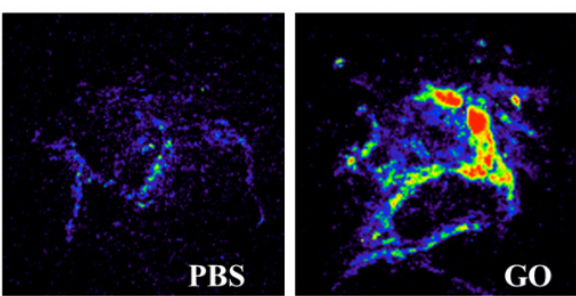

high
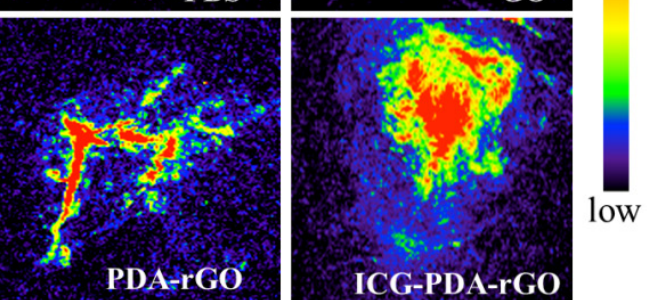

Figure 6. In vivo PA imaging of ICG-PDA-rGO. (a) Schematic illustration of i.t. injected of ICG-PDA-rGO. (b) In vivo PA imaging of tumor treated with PBS, GO, PDA-rGO and ICG-PDA-rGO. (C) Statistics of mean PA intensity of the samples measured from in vivo PA imaging. Error bars were taken from three parallel experiments. $(*)$ P $<0.05$, $(* *) \mathrm{p}<0.01$.

\section{In vivo PTT}

Based on the PA imaging in vivo and PTT in vitro, we expected to apply ICG-PDA-rGO for imaging-guided cancer PTT in vivo. 4T1 breast subcutaneous and orthotopic tumor models in mice were used to evaluate the therapuetic efficiency. PBS, GO, PDA-rGO and ICG-PDA-rGO were intratumorally injected into $4 \mathrm{~T} 1$ breast subcutaneous tumor model in mice when the tumor size grew to 50 $\mathrm{mm}^{3}$, respectively. Then, all mice were irradiated at the tumor site with a NIR laser $\left(808 \mathrm{~nm}, 0.6 \mathrm{~W} / \mathrm{cm}^{2}\right)$ for $5 \mathrm{~min}$. The real-time temperature change of mice was analyzed using an infrared thermal camera, which provided an effective tool for monitoring the treatment process. As shown in Figure 7a, after the 5 min of NIR laser irradiation, the tumors treated with PBS, GO and PDA-rGO exhibited moderate increase to $29.9,39.9$ and $44.4^{\circ} \mathrm{C}$, respectively. While the temperature of tumor treated with ICG-PDA-rGO increased rapidly to $54.6^{\circ} \mathrm{C}$, which was high enough to completely ablate the malignant cells. The histopathological analysis was carried out to illustrate typical thermal damage in the tumors. As shown in Figure $7 b$, in PBS and GO-treated groups, there was 
no obvious tumor necrosis in histological section. On the contrary, some karyolysis and sporadic necrotic was observed in PDA-rGO-treated group. Apparent extensive cancer necrosis occurred with ICG-PDA-rGO-treated group, such as coagulative necrosis, pyknosis and karyolysis. The results indicated that PTT treatment mediated by ICG-PDA-rGO plus single NIR laser could significantly improve the therapeutic efficiency. In order to further investigate the antitumor efficiency of ICG-PDA-rGO in mice bearing 4T1, the growth rate of tumors was monitored every 3 days after treatments. The represent mice photos reflecting the tumor size change showed in Figure S2. As shown in Figure 7c, the mice with GO-treated showed little therapeutic efficacy in comparison with the control group. PDA-rGO-treated group had slow tumor growth rates, and tumor suppression was incomplete. On the contrary, it was worthy to note that a complete remission of tumors was observed in ICG-PDA-rGO treated group and there was no tumor recurrence with $100 \%$ of survival rate on day 40 (Figure $7 \mathrm{~d}$ ). During the treatments, the body weight did not obviously change, which indicated the treatments were safe and nontoxic (Figure S3).

Next, we further evaluate the PTT mediated by ICG-PDA-rGO in 4T1 orthotopic tumor model. The breast tumor grew on the mammary fat pad of mice, keeping a distance from the surface skin. As shown in Figure 8a, using a NIR laser irradiation, the temperature of tumor increased to $53.3{ }^{\circ} \mathrm{C}$ in mice treated with ICG-PDA-rGO within $5 \mathrm{~min}$, which was already sufficient to induce irreversible tumor
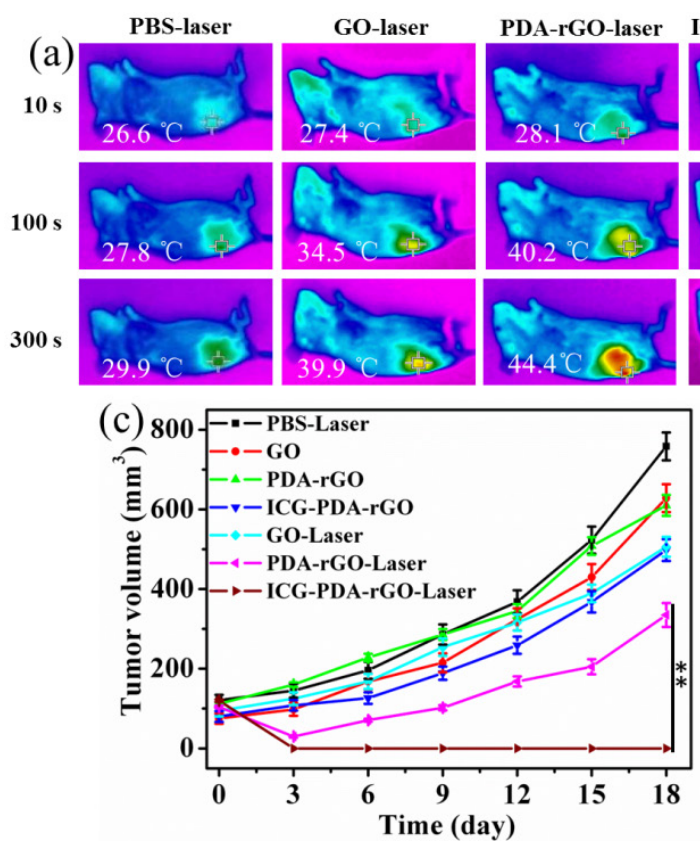

damage. However, other groups did not show a high temperature. As shown in Figure 8b, the tumor was significantly inhibited by ICG-PDA-rGO after laser irradiation, which was in accordance with previous results in subcutaneous tumor model (Figure S4). Similarly, the survival rate of ICG-PDA-rGO PTT treated group were $100 \%$ after 40 days post-treatment (Figure 8c), and the body weight did not obviously change (Figure $8 \mathrm{~d}$ ). The results indicated that the ICG-PDA-rGO could also be successfully applied for tumor PTT in breast tumors with orthotopic mice models.

\section{Biological toxicity assessment}

The ideal theranostic nanoagents should be nontoxic or low-toxic to organs.[40] We have verified that the ICG-PDA-rGO showed no obvious cytotoxicity in normal cell line or 4T1 cells. Furthermore, we investigated the potential biological toxicity, histological and blood analysis in mice. The H\&E staining images of major organs collected from ICG-PDA-rGO treated group, suggesting neither obvious damage nor inflammation was observed compared to the control group, which indicated there was no distinct side effect to the treated mice in vivo based on ICG-PDA-rGO PTT treatment (Figure 9). All blood panel parameters in the ICG-PDA-rGO PTT treated mice were normal, while the other groups showed slight or mild variation (Table S1). These results indicated that the ICG-PDA-rGO possessed low cytotoxicity, and being suitable for cancer photothermal theranostics.
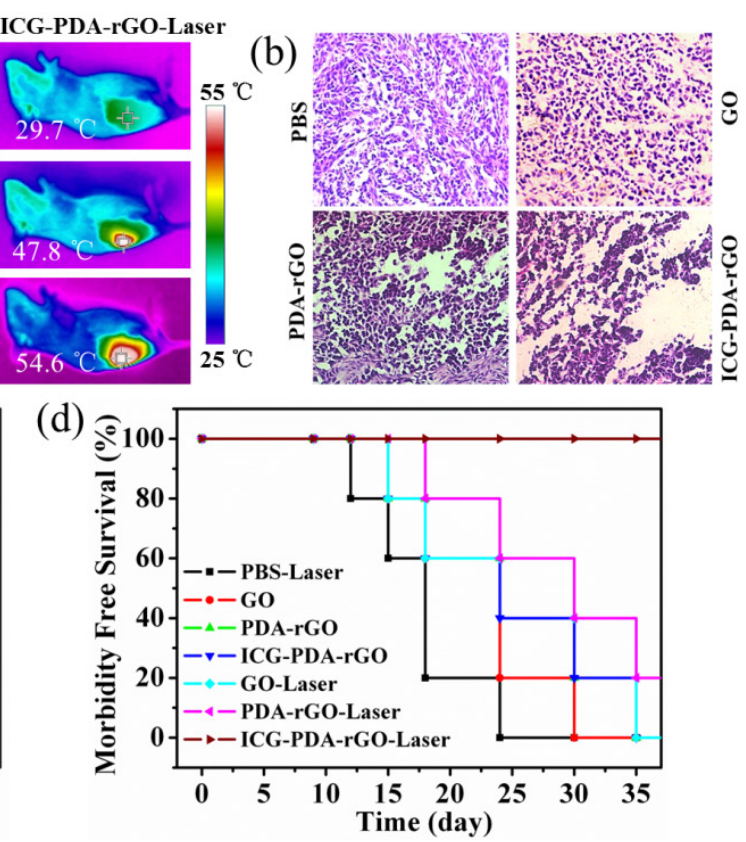

Figure 7. In vivo cancer PTT in xenograft mice model with $4 \mathrm{~T} 1$ breast cancer. (a) Thermal images of $4 \mathrm{~T} 1$ tumor-bearing mice exposed to $808 \mathrm{~nm}$ laser $\left(0.6 \mathrm{~W} / \mathrm{cm}^{2}\right)$ after i.t. injection of PBS, GO, PDA-rGO and ICG-PDA-rGO, respectively. (b) H\&E-stained images of tumor sections collected from different treated groups after $5 \mathrm{~h}$ treatment. (c) Tumor growth curves of different groups of $4 \mathrm{~T} 1$ tumor-bearing mice. (d) Survival rates of mice bearing $4 \mathrm{~T} 1$ tumors after various treatments. $(* *) \mathrm{P}<0.01$. 

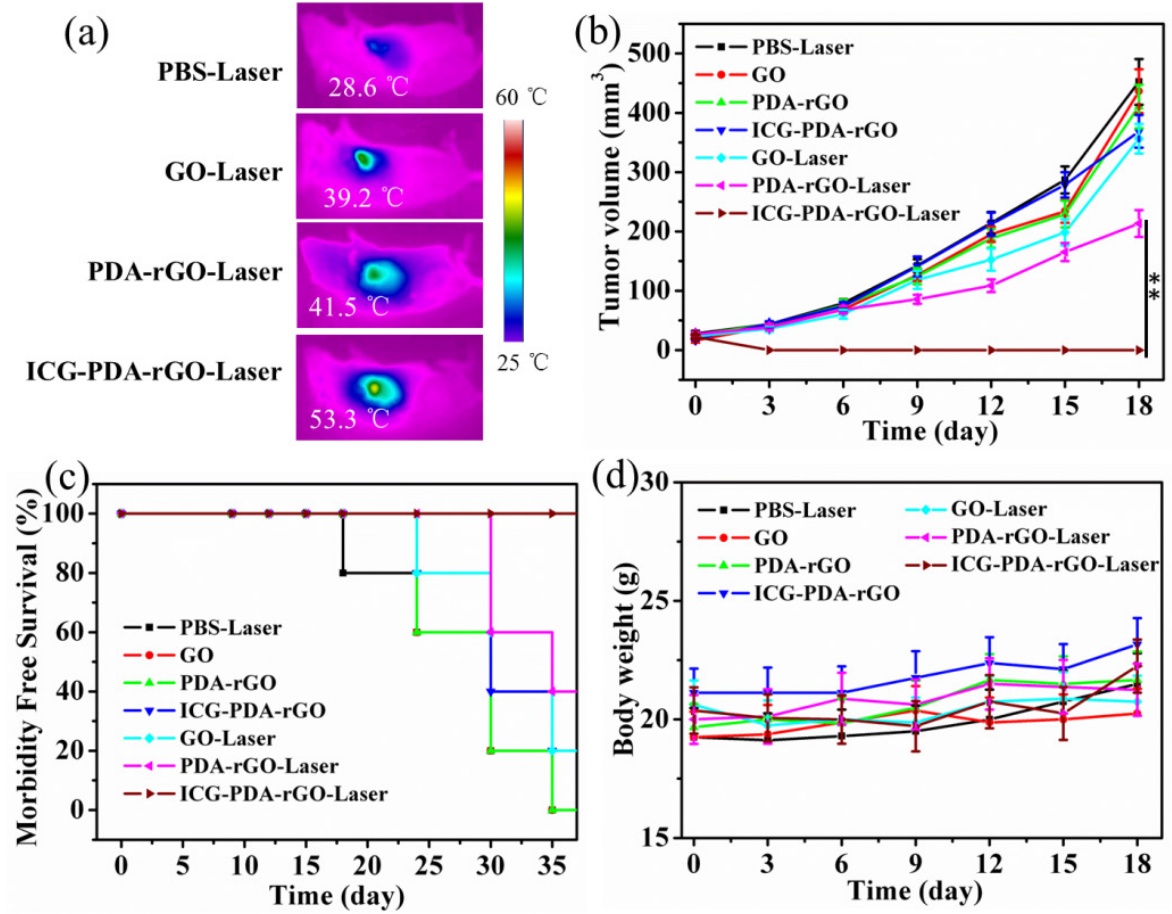

Figure 8. In vivo cancer PTT in orthotopic mice model. (a) Thermal images of $4 \mathrm{~T} 1$ tumor-bearing mice exposed to $808 \mathrm{~nm}$ laser $\left(0.6 \mathrm{~W} / \mathrm{cm}^{2}\right)$ after intratumor injection of PBS, GO, PDA-rGO and ICG-PDA-rGO, respectively. (b) Tumor growth curves of different groups of 4T1 tumor-bearing mice. (c) Survival rates of mice bearing 4T1 tumors after various treatments. (d) Body weights were measured during 18 day evaluation period in mice with different treatments. $(* *) p<0.01$.

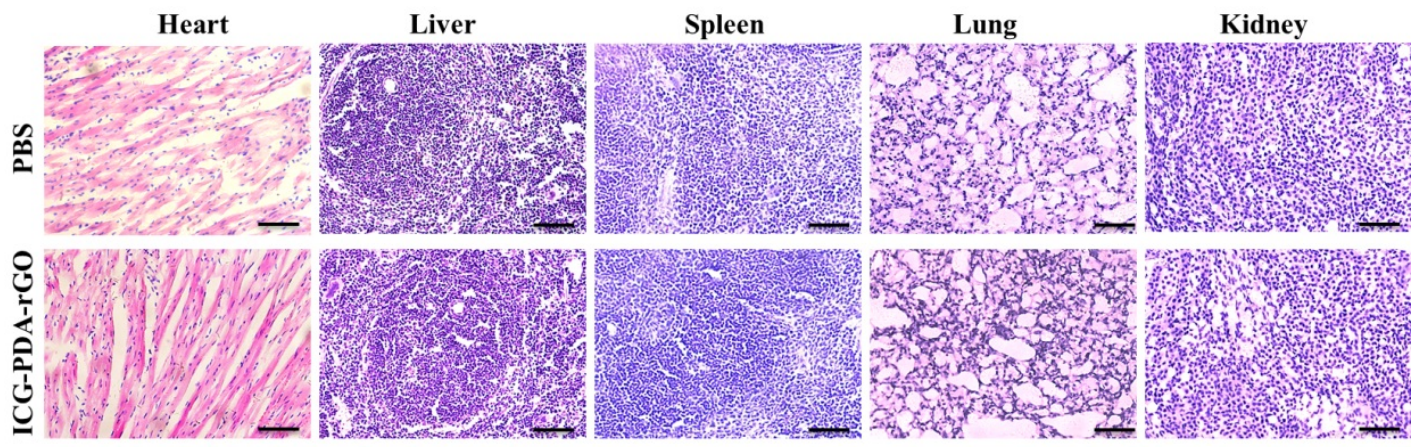

Figure 9. Representative H\&E stained images of major organs including the heart, liver, spleen, lung and kidney collected from the PBS injected mice and ICG-PDA-rGO injected mice. The dose of ICG-PDA-rGO was $2 \mathrm{mg} / \mathrm{kg}$. These images were obtained under a light microscope.

\section{Conclusions}

In summary, we have developed a new GO derivatives, ICG-PDA-rGO, for amplifying PA and PTT effects for cancer phototheranostics. The prepared PDA-rGO was able to improve the solubility and stability of rGO, efficiently absorb ICG molecules, and enhance the optical absorption in NIR wavelength. The in vitro, in vivo PA and PTT effects of ICG-PDA-rGO were significantly promoted compared to that of PDA-rGO. The ICG-PDA-rGO-mediated PTT treatments efficiently improved the anticancer effect, leading to superior tumor eradication in $4 \mathrm{~T} 1$ breast subcutaneous and orthotopic tumor models. The further improved the novel phototheranostic platform with reduced the lateral dimension would be a promising applications in highly sensitive PA imaging and efficient cancer phototherapy, it was expected to have a prospective potential in clinical translation.

\section{Supplementary Material}

Figures S1-S4 and Table S1.

http://www.thno.org/v06p1043s1.pdf

\section{Acknowledgements}

This work was supported by the Major State Basic Research Development Program of China (973 Program) (2015CB755500), Natural Science Foundation of China $(31571013,81301272,81571745$, 81401521， 21203237), Key International S\&T 
Cooperation Project (2015DFH50230), Instrument Developing Project of the CAS (YZ201439), the Science and Technology Innovation Fund of Shenzhen (JCYJ20150401145529015 and KQCX201405211150454 47), Shenzhen Bioactive Materials Engineering Lab for Medicine ([2013]154), Key International S\&T Cooperation Project (2015DFH50230), Instrument Developing Project of CAS (YZ201439), SIAT Innovation Program for Excellent Young Researchers (201301).

\section{Competing Interests}

The authors have declared that no competing interest exists.

\section{References}

1. Cheng L, Liu J, Gu X, Gong H, Shi X, Liu T, et al. PEGylated WS(2) nanosheets as a multifunctional theranostic agent for in vivo dual-modal $\mathrm{CT} /$ photoacoustic imaging guided photothermal therapy. Adv Mater. 2014;26:1886-93.

2. Liu J, Zheng X, Yan L, Zhou L, Tian G, Yin W, et al. Bismuth sulfide nanorods as a precision nanomedicine for in vivo multimodal imaging-guided photothermal therapy of tumor. ACS Nano. 2015;9:696-707.

3. Liang X, Li Y, Li X, Jing L, Deng Z, Yue X, et al. PEGylated polypyrrole nanoparticles conjugating gadolinium chelates for dual-modal MRI/photoacoustic imaging guided photothermal therapy of cancer. Adv Fun Mater. 2015;25:1451-62

4. Wang LV, Hu S. Photoacoustic tomography: in vivo imaging from organelles to organs. Science. 2012;335:1458-62.

5. Nie L, Huang P, Li W, Yan X, Jin A, Wang Z, et al. Early-stage imaging of nanocarrier-enhanced chemotherapy response in living subjects by scalable photoacoustic microscopy. ACS Nano. 2014;8:12141-50.

6. Li J, Arnal B, Wei CW, Shang J, Nguyen TM, O'Donnell M, et al. Magneto-optical nanoparticles for cyclic magnetomotive photoacoustic imaging. ACS Nano. 2015;9:1964-76.

7. Cheng L, Wang C, Feng L, Yang K, Liu Z. Functional nanomaterials for phototherapies of cancer. Chem Rev. 2014;114:10869-939.

8. Song KH, Kim C, Cobley CM, Xia Y, Wang LV. Near-infrared gold nanocages as a new class of tracers for photoacoustic sentinel lymph node mapping on a rat model. Nano Lett. 2009:9:183-8.

9. Huang X, Jain PK, El-Sayed IH, El-Sayed MA. Plasmonic photothermal therapy (PPTT) using gold nanoparticles. Lasers Med Sci. 2008;23:217-28.

10. Hu J, Zhu X, Li H, Zhao Z, Chi X, Huang G. Theranostic Au cubic nano-aggregates as potential photoacoustic contrast and photothermal therapeutic agents. Theranostics. 2014;4:534-45.

11. Zhou M, Zhang R, Huang M, Lu W, Song S, Melancon MP, et al. A chelator-free multifunctional $\left[{ }^{64} \mathrm{Cu}\right] \mathrm{CuS}$ nanoparticle platform for simultaneous micro-PET/CT imaging and photothermal ablation therapy. J Am Chem Soc. 2010;132:15351-8.

12. Ku G, Zhou M, Song S, Huang Q, Hazle J, Li C. Copper sulfide nanoparticles as a new class of photoacoustic contrast agent for deep tissue imaging at 1064 nm. ACS Nano. 2012;6:7489-96.

13. Liu T, Shi S, Liang C, Shen S, Cheng L, Wang C, et al. Iron oxide decorated $\mathrm{MoS}_{2}$ nanosheets with double PEGylation for chelator-free radiolabeling and multimodal imaging guided photothermal therapy. ACS Nano. 2015;9:950-60.

14. Chou SS, Kaehr B, Kim J, Foley BM, De M, Hopkins PE, et al. Chemically exfoliated $\mathrm{MoS}_{2}$ as near-infrared photothermal agents. Angew Chem Int Ed Engl. 2013;52:4160-4.

15. Gong H, Peng R, Liu Z. Carbon nanotubes for biomedical imaging: the recent advances. Adv Drug Deliv Rev. 2013;65:1951-63.

16. Yang K, Feng L, Shi X, Liu Z. Nano-graphene in biomedicine: theranostic applications. Chem Soc Rev. 2013;42:530-47.

17. $\mathrm{Pu} \mathrm{K}$, Shuhendler AJ, Jokerst JV, Mei J, Gambhir SS, Bao Z, et al. Semiconducting polymer nanoparticles as photoacoustic molecular imaging probes in living mice. Nat Nanotechnol. 2014;9:233-9.

18. Zha $Z$, Yue $X$, Ren $Q$, Dai Z. Uniform polypyrrole nanoparticles with high photothermal conversion efficiency for photothermal ablation of cancer cells. Adv Mater. 2013;25:777-82

19. Cheng L, Yang K, Chen Q, Liu Z. Organic stealth nanoparticles for highly effective in vivo near-infrared photothermal therapy of cancer. ACS Nano. 2012:6:5605-13.

20. Mao HY, Laurent S, Chen W, Akhavan O, Imani M, Ashkarran AA, et al. Graphene: promises, facts, opportunities, and challenges in nanomedicine. Chem Rev. 2013;113:3407-24.

21. Liu Z, Robinson JT, Sun X, Dai H. PEGylated nanographene oxide for delivery of water-insoluble cancer drugs. J Am Chem Soc. 2008;130:10876-7.
22. Zhang L, Lu Z, Zhao Q, Huang J, Shen H, Zhang Z. Enhanced chemotherapy efficacy by sequential delivery of siRNA and anticancer drugs using PEI-grafted graphene oxide. Small. 2011;7:460-4.

23. Rong P, Yang K, Srivastan A, Kiesewetter DO, Yue X, Wang F. Photosensitizer loaded nano-graphene for multimodality imaging guided tumor photodynamic therapy. Theranostics. 2014;4:229-39.

24. Shen H, Zhang L, Liu M, Zhang Z. Biomedical applications of graphene. Theranostics. 2012;2:283-94.

25. Feng L, Zhang S, Liu Z. Graphene based gene transfection. Nanoscale. 2011;3:1252-7.

26. Yang K, Hu L, Ma X, Ye S, Cheng L, Shi X, et al. Multimodal imaging guided photothermal therapy using functionalized graphene nanosheets anchored with magnetic nanoparticles. Adv Mater. 2012;24:1868-72.

27. Sheng Z, Song L, Zheng J, Hu D, He M, Zheng M, et al. Protein-assisted fabrication of nano-reduced graphene oxide for combined in vivo photoacoustic imaging and photothermal therapy. Biomaterials. 2013;3:5236-43.

28. Yang K, Wan J, Zhang S, Tian B, Zhang Y, Liu Z. The influence of surface chemistry and size of nanoscale graphene oxide on photothermal therapy of cancer using ultra-low laser power. Biomaterials. 2012;33:2206-14.

29. Moon H, Kumar D, Kim H, Sim C, Chang JH, Kim JM, et al. Amplified photoacoustic performance and enhanced photothermal stability of reduced graphene oxide coated gold nanorods for sensitive photoacoustic imaging. ACS Nano. 2015;9:2711-9.

30. Song J, Yang X, Jacobson O, Lin L, Huang P, Niu G et al. Sequential drug release and enhanced photothermal and photoacoustic effect of hybrid reduced graphene oxide-loaded ultrasmall gold nanorod vesicles for cancer therapy. ACS Nano. 2015:9:9199-209.

31. Lin LS, Cong ZX, Cao JB, Ke KM, Peng QL, Gao J, et al. Multifunctional $\mathrm{Fe}_{3} \mathrm{O}_{4} @$ polydopamine core-shell nanocomposites for intracellular mRNA detection and imaging-guided photothermal therapy. ACS Nano. 2014;8:3876-83.

32. Sheng Z, Hu D, Xue M, He M, Gong P, Cai L. Indocyanine green nanoparticles for theranostic applications. Nano-Micro Lett. 2013;5:145-50.

33. Sheng $Z, H u$ D, Zheng $M$, Zhao $P$, Liu H, Gao D, et al. Smart human serum albumin-indocyanine green nanoparticles generated by programmed assembly for dual-modal imaging-guided cancer synergistic phototherapy. ACS Nano. 2014;8:12310-22.

34. Xu L, Yang W, Neoh KG, Kang ET, Fu G. Polydopamine and its derivative materials: synthesis and promising applications in energy, environmental, and biomedical fields. Chem Rev. 2014;114:5057-115.

35. Liu Y, Ai K, Lu L, Xu L, Yang W, Neoh KG, et al. Dopamine-induced reduction and functionalization of graphene oxide nanosheets. Macromolecules. 2010;43:8336-39.

36. Robinson JT, Tabakman SM, Liang $Y$, Wang $H$, Casalongue HS, Vinh $D$, et al. Ultrasmall reduced graphene oxide with high near-infrared absorbance for photothermal therapy. J Am Chem Soc. 2011;133:6825-31.

37. Maslov K, Wang LV. Photoacoustic imaging of biological tissue with intensity-modulated continuous-wave laser. J Biomed Opt. 2008;13:024006.

38. Goldberg EP, Hadba AR, Almond BA, Marotta J S. Intratumoral cancer chemotherapy and immunotherapy: opportunities for nonsystemic preoperative drug delivery. J Pharm Pharmacol. 2002:54:159-80.

39. Zheng M, Yue C, Ma Y, Gong P, Zhao P, Zheng C, et al. Single-step assembly of DOX/ICG loaded lipid--polymer nanoparticles for highly effective chemo-photothermal combination therapy. ACS Nano. 2013;7:2056-67.

40. Mitragotri S, Anderson DG, Chen X, Chow EK, Ho D, Kabanov AV, et al. Accelerating the translation of nanomaterials in biomedicine. ACS Nano. 2015;9:6644-54. 